\title{
Ovarian small-cell carcinoma hypercalcemic type successfully treated: a case report and literature review
}

This article was published in the following Dove Press journal:

OncoTargets and Therapy

II March 2016

Number of times this article has been viewed

\author{
Jian-jun Wangl,* \\ Qi Liu',* \\ Nan Wu' \\ Xiao-die Zhou' \\ Hai Wang' \\ Xuan Wang' \\ Pin Tu' \\ Xiao-jun Zhou' \\ Qun-li Shi' \\ 'Department of Pathology, \\ ${ }^{2}$ Department of Obstetrics and \\ Gynecology, Jinling Hospital, Medical \\ School of Nanjing University, Nanjing \\ University, Nanjing, Jiangsu, People's \\ Republic of China \\ *These authors contributed equally \\ to this work
}

Correspondence: Qun-li Shi

Department of Pathology, Jinling Hospital, Medical School of Nanjing University, Nanjing University, 305 East Zhongshan Road, Nanjing, Jiangsu 210002, People's Republic of China

Tel +860258086 |29|
Email shiqunli2005@aliyun.com

\begin{abstract}
Ovarian small-cell carcinoma hypercalcemic type (OSCCHT) is a relatively rare and highly fatal gynecological malignancy. Prognosis is generally poor, and no treatment guidelines are offered. Here, we report a case of OSCCHT successfully treated by complete excision and a postoperative chemotherapy scheme of carboplatin and paclitaxel. A 29-year-old female with with pelvic mass and significantly increased serum calcium $(4.90 \mathrm{mmol} / \mathrm{L})$ was referred to our hospital on August 22, 2013. Abdominal ultrasonography and computed tomography revealed a pelvic nonhomogeneous echo of a $113 \times 102 \mathrm{~mm}$ mass, possibly coming from the adnexa of the uterus. Preoperative examinations indicated high levels of serum calcium and relevant acute renal dysfunction; hence, continuous renal replacement therapy was performed until all tests reached minimum operation requirements. Interestingly, after excision, serum calcium levels decreased rapidly and therefore, extra calcium had to be taken in order to take the level back up to normal. The patient was diagnosed with OSCCHT based on the clinical data and pathological examinations. After six cycles of chemotherapy, the patient was in a good condition and on follow-up there were no signs of recurrence.
\end{abstract}

Keywords: small-cell carcinoma, hypercalcemic type, treatment, successful, ovary

\section{Introduction}

Primary ovarian small-cell carcinoma is a rare and aggressive gynecological malignancy with a tendency of early distant metastases and a low 5-year survival rate. There are two types of ovarian small-cell carcinomas: hypercalcemic and pulmonary. Ovarian small-cell carcinoma hypercalcemic type (OSCCHT), first described in 1975 by Scully, ${ }^{1}$ mainly occurs in young females and is often accompanied by paraneoplastic syndrome of hypercalcemia.

OSCCHT has no specific symptoms and is often revealed as a nodular or lobulated neoplasm in one of the adnexa of the uterus. The tumor is thought to be a highly fatal neoplasm and survival prognosis is poor. The treatment of OSCCHT is challenging, and no mature therapy guidelines are offered. Here, we report a case of OSCCHT associated with severe hypercalcemia and acute renal dysfunction which was successfully treated by complete tumor excision and postoperative chemotherapy.

\section{Case report}

On August 22, 2013, a 29-year-old female presented to a local hospital with inappetence and significant weight loss (about $10 \mathrm{~kg}$ ) over 1 month. Blood tests showed increased levels of alanine transaminase (58.9 IU/L) and alkaline phosphatase (133 IU/L) and a low serum potassium level $(3.3 \mathrm{mmol} / \mathrm{L})$. The patient was then referred to another (c)
hereby accept the Terms. Non-commercial uses of the work are permitted without any further permission from Dove Medical Press Limited, provided the work is properly attributed. For permission hereby accept the Terms. Non-commercial uses of the work are permitted without any further permission from Dove Medis
for commercial use of this work, please see paragraphs 4.2 and 5 of our Terms (https://www.dovepress.com/terms.php). 
hospital for further examinations, and renal function tests showed an increase in urea nitrogen $(9.11 \mathrm{mmol} / \mathrm{L})$, serum creatinine $(202.7 \mu \mathrm{mol} / \mathrm{L})$, and uric acid $(678.7 \mu \mathrm{mol} / \mathrm{L})$. Abdominal ultrasonography revealed a pelvic nonhomogeneous echo of a $113 \times 102 \mathrm{~mm}$ mass, and pelvic computed tomography indicated that the pelvic mass possibly came from adnexa of the uterus. No increased carcinoembryonic antigen or carbohydrate antigen (CA)-199 was noted. The patient was transferred to Jinling Hospital for further diagnosis and treatment on September 3, 2013 (day 1).

Laboratory findings on admission were as follows: serum calciumlevels at $4.90 \mathrm{mmol} / \mathrm{L}$ (normal, 2.03-2.54 mmol/L), parathyroid hormone at $1.1 \mathrm{pmol} / \mathrm{L}$ (normal, 1.6-9.3 pmol/L), urea nitrogen at $13.3 \mathrm{mmol} / \mathrm{L}$ (normal, 2.86-7.14 mmol/L), serum creatinine at $219 \mu \mathrm{mol} / \mathrm{L}$ (normal, $44-133 \mu \mathrm{mol} / \mathrm{L}$ ), uric acid at $678.7 \mu \mathrm{mol} / \mathrm{L}$ (normal, 178.4-297.4 $\mu \mathrm{mol}$ ), CA125 at $43.60 \mathrm{U} / \mathrm{mL}$ (normal, $<35 \mathrm{U} / \mathrm{mL}$ ), and lactate dehydrogenase at $384 \mathrm{U} / \mathrm{L}$ (normal, 100-300 U/L).

During a gynecological examination a big, hard, fixed, and non-tender mass right in front of the uterus was observed. Pelvic magnetic resonance imaging revealed a less uniform mass signal the size of $110 \times 116 \times 125 \mathrm{~mm}$, with visible low nodular signal inside the tumor and a less clear boundary between the conjunction of the uterus and the tumor (Figure 1). No other tumor was found in the colon or bladder. Thoracic and abdominal radiography showed nothing unusual.

To study the reason behind the abnormal hypercalcemia, thyroid ultrasonography and computed tomography were performed, but no tumor was found in the thyroid or parathyroid. Thus, hypercalcemia might have been caused by the malignant tumor, and increasingly high calcium levels might have been the cause of acute renal dysfunction. Therefore, continuous renal replacement therapy was performed on day 3 . An emergent operation was performed once urea nitrogen and serum creatinine tests were within normal limits after two rounds of hemodialysis on day 4.

During surgery, no adhesion between abdominal viscera was inspected. The right ovary increased approximately to the size of $12 \times 10 \times 10 \mathrm{~cm}$ with a hard, uneven, and vascular surface (Figure 2). The right fallopian tube, uterine, and left adnexa were all within the normal range. Preoperative function of blood coagulation showed a thrombin time of 26.6 seconds, fibrinogen level of $419 \mathrm{mg} / \mathrm{dL}$, and a partially activated thromboplastin time of 58.3 seconds, which were all beyond normal limits. Examinations of frozen sections revealed a malignant tumor. Considering these facts: hypercalcemia, renal dysfunction, and abnormal dysfunction of coagulation, unilateral adnexectomy was performed. After surgery, serum calcium decreased rapidly, and on day 8 calcium levels had to be brought back up to normal levels by taking extra calcium (Figure 3). Microscopically, the tumor cells were arranged in a solid distribution, nodular, and pseudoglandular cavity patterns, with medium to large cells, eosinophilic cytoplasm, round or oval vesicular nucleus, increased karyoplasmic ratio, and pathologic mitosis (Figure 4A and B). Immunochemistry showed EMA (+), CK (+), C-erbB-2 (1+), CA125 (focal +), and $\mathrm{Ki}-67$ proliferation index labeling $\sim 60 \%$, while estrogen (ER), progesterone (PR), inhibin, CD99, alpha fetoprotein (ALP), placental - type alkaline phosphatase (PLAP), CD30, and CD20 were not expressed. Kras mutation was not detected, and no tumor cells were found in ascites cytological examination. The most striking ultrastructural features revealed no neuroendocrine granules, while visible inserted basement membrane and cell junction could be seen, suggesting tumors of an epithelial origin (Figure 4C). All in all, the final diagnosis of OSCCHT was made.

On day 9, the patient received a carboplatin and paclitaxel chemotherapy scheme when the examination of the liver and renal functions were within the normal range. On October
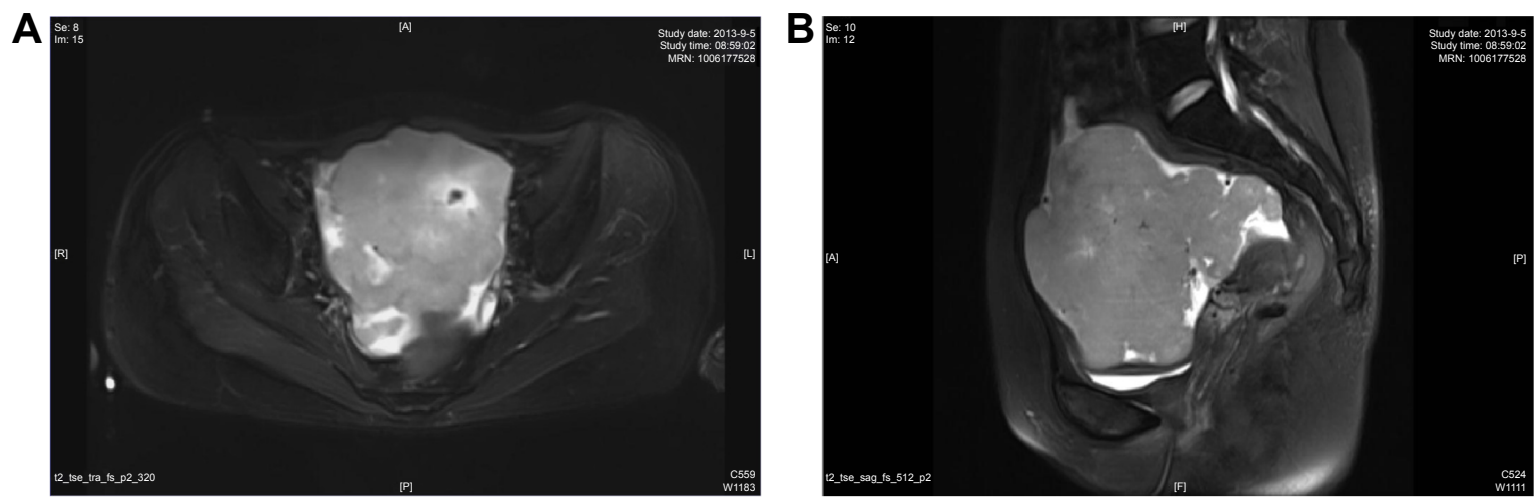

Figure I Magnetic resonance imaging of the tumor on admission.

Notes: (A) Magnetic resonance imaging (T2) displayed a less uniform mass signal with a size of I I0 1 I $16 \times 125$ mm, with visible low nodular signal inside the tumor. (B) Magnetic resonance imaging (T2) showed a tumor with less clear boundary between the conjunction of the uterus and the tumor oppressing the uterus and other pelvic organs. 


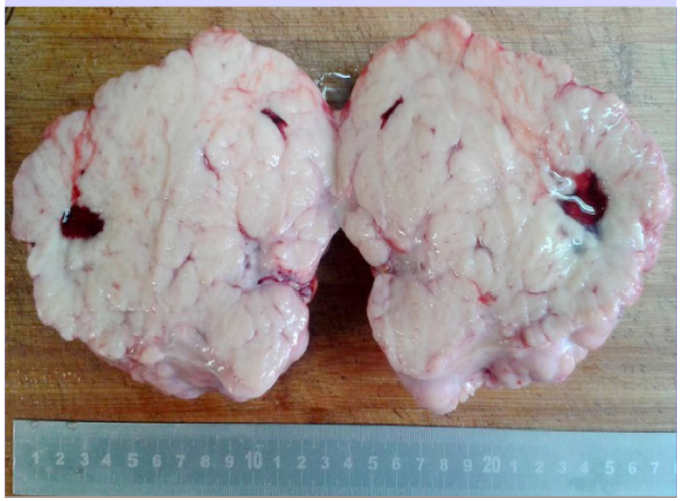

Figure 2 Pathological characteristics.

Notes: The tumor displayed a nodular appearance with a diameter of $\sim 12 \mathrm{~cm}$, and cross-section showed white appearance and medium texture, with a loculus of $1.2 \mathrm{~cm}$ in diameter filled with a pale yellow fluid.

25 , the patient received extensive excision, and postoperative pathology found para-aortic lymph node metastasis (1/13). The patient received five more rounds of chemotherapy on October 3, October 30, November 25, December 21, 2013, and January 12, 2014. The patient was in a good clinical condition, and serum calcium and urea nitrogen were found to be at normal levels during regular follow-ups (Figure 3), with no recurrence or metastasis by the latest follow-up on September 20, 2015.

\section{Discussion}

OSCCHT is considered as a highly aggressive variant of ovarian malignancies, associated with an extremely unfavorable prognosis. OSCCHT mainly occurs in young females (mean age: 22 years), and $75 \%$ of patients die within 1 or 2 years. ${ }^{2}$ OSCCHT has no specific clinical manifestations, and is mainly presented as unilateral adnexal masses of equal incidence to the left or right side. Therefore, definitive preoperative diagnosis of OSCCHT is frequently difficult and pathological examination remains the gold standard for its diagnosis. Several reports of OSCCHT cases were

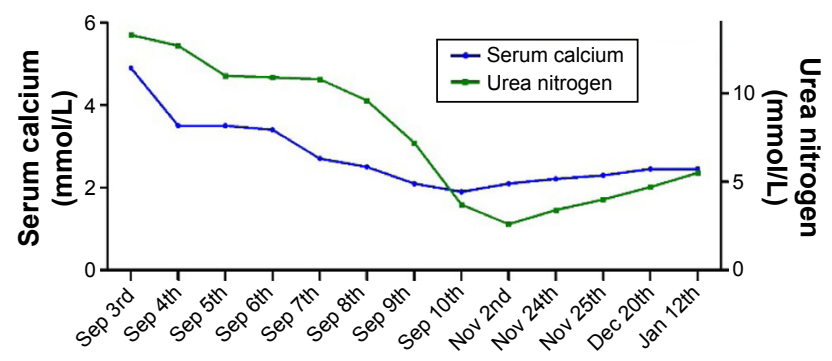

Figure 3 The changing of serum calcium and urea nitrogen levels. Notes: The serum calcium levels sharply decreased after complete excision (on September 6, 2013); hence, extra calcium had to be taken to increase levels back to normal. The urea nitrogen levels indicated revised renal function. found in family members, suggesting that OSCCHT could be heritable. ${ }^{2-4}$ The tumors were generally large in size, with an average diameter of $15 \mathrm{~cm}$, and had a nodular or lobulated appearance with mostly solid or partially cystic sections accompanied by visible hemorrhage and necrosis. In two-thirds of cases, OSCCHT was often presented with paraneoplastic syndrome of hypercalcemia, while only $10 \%$ of cases had hypercalcemia symptoms. ${ }^{5}$ It is difficult to explain this phenomenon as the mechanism of hypercalcemia is not understood clearly, but it is generally believed that hypercalcemia is caused by tumor cells unlike the mechanism of neuroendocrine tumors. Related literature reported variable serum calcium levels of 2.43-4.80 mmol/L, with an average of $3.68 \mathrm{mmol} / \mathrm{L}^{2,4,6-11} \mathrm{In}$ the present case, this patient had a much higher level of serum calcium of $4.90 \mathrm{mmol} / \mathrm{L}$ preoperative than what other literature reported, while postoperative serum calcium levels decreased to $1.9 \mathrm{mmol} / \mathrm{L}$. Hence, on day 8, calcium levels had to be brought back up to the normal level, and it then stayed normal during the followups, which indicated that increased serum calcium levels were caused by tumor cells. After surgery, serum calcium levels returned to normal, reflecting that serum calcium levels corresponds with surgical treatment which might be a useful means of monitoring the disease. ${ }^{5}$ As serum calcium levels decreased, urea nitrogen gradually returned to normal levels, which indicated that increased serum calcium levels might induce acute renal dysfunction and, more importantly, indirectly proved that high levels of serum calcium were caused by the tumor. Some studies recommended that CA-125 could be a very useful tumor marker, which was elevated in approximately $75 \%$ of patients at diagnosis, ${ }^{12}$ and CA-125 levels were slightly high in the present case also.

Treatment included surgery, radiotherapy, and chemotherapy while there was no unified view on which one should be given priority, and it was so poorly differentiated that no experienced adjuvant chemotherapy guidelines were offered. OSCCHT is an ovarian epithelial cancer type in nature, and carboplatin and paclitaxel regimens are commonly used as the first-line chemotherapeutic regimen for ovarian epithelial cancers. Woopen et $\mathrm{al}^{8}$ reported on three cases using six cycles of carboplatin and paclitaxel as first-line therapy and reported no recurrence at the time of publishing, which is the same method and results as to what $\mathrm{Wynn}$ et $\mathrm{al}^{9}$ and the present case had. Carboplatin and paclitaxel regimens were initially used in the presented case without any progression. The situation was well stabilized and the patient's condition improved over the following several cycles. However, a previous study reported only limited efficacy of both first line and salvage therapy in treating OSCCHT. ${ }^{13}$ In Harrison et al's case report, ${ }^{14}$ of the 

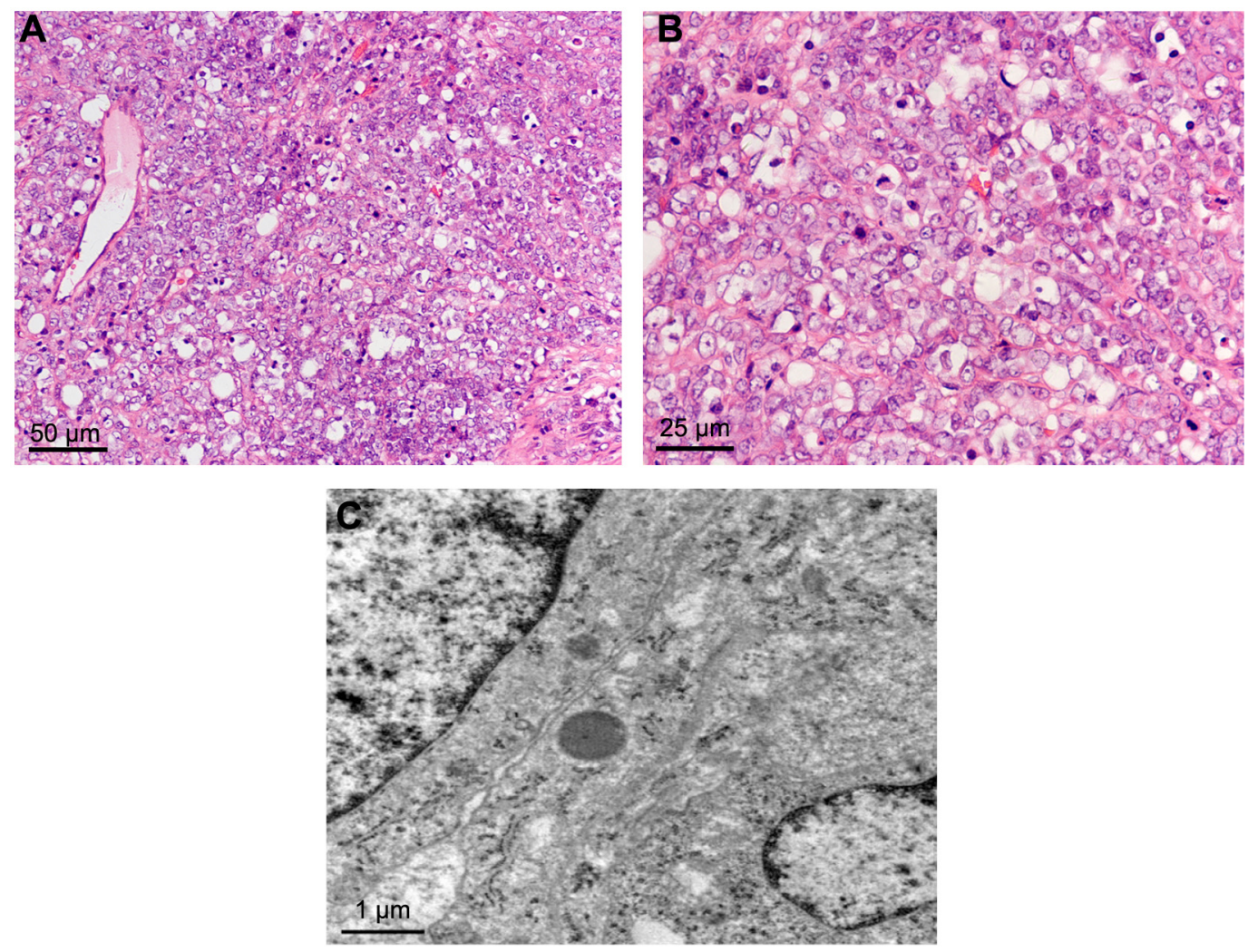

Figure 4 Pathological characteristics.

Notes: (A) The tumor cells were arranged in a solid distribution and nodules splitted by fiber bundles with scattered pseudoglandular or follicular-like cavities (H\&E staining; magnification $\times 200$ ). (B) The tumor had medium to large cells, eosinophilic cytoplasm, and round or oval vesicular nucleus, with increased karyoplasmic ratio and pathologic mitosis (H\&E staining; magnification $\times 400$ ). (C) Electron microscope revealed the cell junction and inserted basement membrane without neurosecretory granules.

Abbreviation: H\&E, hematoxylin and eosin staining.

four patients who received carboplatin and paclitaxel, two had disease progression during treatment and two progressed within 3 months of chemotherapy. Another reported regimen of VPCBAE (vinblastine, cisplatin, cyclophosphamide, bleomycin, doxorubicin, etoposide), has been used in the treatment of OSCCHT with varying success. ${ }^{15,16}$ Wallbillich et $\mathrm{al}^{17}$ presented a case series of three patients with OSCCHT with unilateral salpingo-oophorectomy followed by six cycles of chemotherapy with VPCBAE without evidence of recurrence. Walker et a ${ }^{18}$ reported that advanced stage III OSCCHT patients who received VPCBAE therapy had been disease-free for more than 10 years. Bakhru et a $\mathrm{l}^{19}$ reported the case of a 14-year-old female with no evidence of disease after treatment with four cycles of VPBCAE, however, after 3 weeks there was an appearance of a large pelvic mass with metastases, and the patient was dead within 2 months. OSCCHT was initially treated like small-cell lung cancer as presented in the Gynaecological Cancer Intergroup trial, ${ }^{14}$ which established the use of cisplatin and etoposide for treating this rare subtype of ovarian cancer. ${ }^{8}$ Harrison et al ${ }^{14}$ reported that five stage I patients were alive and disease-free at a median follow-up of 50 months, noting that all long-term survivors received cisplatin and etoposide. Most of them received radiotherapy either sequentially or concurrently, while it was not possible to say that it was superior to carboplatin and paclitaxel as the patients receiving the latter combination had more advanced diseases. In another review, 27 patients received dose-intense PAVEP therapy (cisplatin, adriamycin, VePesid, cyclophosphamide), which was found to be effective and the patients had an overall 5-year survival rate of $49 \%$ and 14 stage III patients were without any sign of disease..$^{20}$

The largest series was reported by Young, ${ }^{5}$ which included 150 patients. Of these patients, $50 \%$ had stage I, $5 \%$ stage II, $43 \%$ stage III, and $1 \%$ stage IV disease. In the report, $33 \%$ of patients with stage IA were alive and disease-free at 5 years, and only $10 \%$ with stage IC and stage II, III, and IV were alive. Furthermore, patients who received adjuvant chemotherapy had better survival in International Federation of Gynecology and Obstetrics stage IA compared with those who had only surgical treatment, while there was no apparent evidence that adjuvant chemotherapy in stage IA could improve the prognosis. ${ }^{5}$ Features associated with a more favorable survival in patients with stage IA tumors included age $>30$ years, a normal preoperative serum calcium level, 
tumor size $<10 \mathrm{~cm}$, localized stage of tumor, and the absence of large cells, while Rebecca et a ${ }^{12}$ thought that tumors with a diameter $>10 \mathrm{~cm}$ were associated with improved survival. Woopen et $\mathrm{al}^{8}$ added that tumor stage appeared to play a significant role in the overall prognosis.

Stephens et $\mathrm{al}^{21}$ analyzed molecular characterization of an OSCCHT patient's sample and found some upregulation or downregulation molecular targets and suggested available potential targeted drugs. Recently, SMARCA4 mutations and SMARCA4 protein loss were identified in OSCCHT, and SMARCA4 (BRG1) loss of expression was thought to be a useful diagnostic marker of OSCCHT in ovarian tumors. ${ }^{22}$ Related data suggested a tumor suppressor role of SMARCA4 and that it might constitute a key therapeutic vulnerability in SMARCA4-deficient cells in the future. ${ }^{23,24}$

\section{Conclusion}

OSCCHT is a rare and aggressive gynecological malignancy and has a poor prognosis. In light of this case report, we think that complete surgical management and postoperative chemotherapy are necessary. As the rarity of the tumor and lack of large series evaluating therapeutic strategies, it is obliged that more case series reports and multicenter analyses are needed to further investigate this rare disease and optimize clinical treatments.

\section{Acknowledgments}

The case report is part of the Ovarian Caricinoma Research Program and it has been approved by the Ethics Committee of Jinling Hospital, Medical School of Nanjing University (number 2015NZKY-013-01) and the Chinese Clinical Trial Register number was ChiCTR-OOC-15006925. This case report is only for medical communication, not for other purposes, and does not include the patient's name and information. The patient agreed to this report being published and signed informed consent.

\section{Disclosure}

The authors report no conflicts of interest in this work.

\section{References}

1. Scully RE. Tumors of the ovary and maldeveloped gonads. Armed Forces Institute Pathol. 1979:153-173.

2. McDonald J, Karabakhtsian R. Small cell carcinoma of the ovary of hypercalcemic type: a case report. J Pediatr Surg. 2012;47(3):588-592.

3. Longy M, Toulouse C, Mage P, Chauvergne J, Trojani M. Familial cluster of ovarian small cell carcinoma: a new Mendelian entity? J Med Genet. 1996;33(4):333-335.

4. Martinez-Borges AR, Petty JK, Hurt G, Stribling JT, Press JZ, Castellino SM. Familial small cell carcinoma of the ovary. Pediatr Blood Cancer. 2009;53(7):1334-1336.
5. Young RH. Small cell carcinoma of the ovary, hypercalcemic type A clinicopathological analysis of 150 cases. Am J Surg Pathol. 1994; 18(11):1102-1116.

6. Popiolek DA, Kumar AR, Mittal K. Large cell variant of small cell carcinoma, hypercalcemic type, of primary peritoneal origin. Gynecol Oncol. 2005;96(1):249-253.

7. Doyle M-A, Malcolm JC. An unusual case of malignancy-related hypercalcemia. Int J Gen Med. 2013;7(7):21-27.

8. Woopen H, Sehouli J, Pietzner K, Darb-Esfahani S, Braicu EI, Fotopoulou C. Clinical experience of young patients with small cell ovarian carcinoma of the hypercalcemic type (OSCCHT). Eur J Obstet Gynecol Reproduct Biol. 2012;165(2):313-317.

9. Wynn D, Everett GD, Boothby RA. Small cell carcinoma of the ovary with hypercalcemia causes severe pancreatitis and altered mental status. Gynecol Oncol. 2004;95(3):716-718.

10. Reed WC. Small cell carcinoma of the ovary with hypercalcemia: report of a case of survival without recurrence 5 years after surgery and chemotherapy. Gynecol Oncol. 1995;56(3):452-455.

11. Zaied S, Gharbi O, Zayene A, et al. Small cell carcinoma of the ovary of hypercalcemic type: a case report. Case Rep Oncol Med. 2012; 2012:3.

12. Rebecca E, Andreas H, Matthias K, Karsten M. Small cell carcinoma of the ovary of the hypercalcaemic type: an analysis of clinical and prognostic aspects of a rare disease on the basis of cases published in the literature. Arch Gynecol. 2011;284(5):1277-1282.

13. Distelmaier F, Calaminus G, Harms D, et al. Ovarian small cell carcinoma of the hypercalcemic type in children and adolescents: a prognostically unfavorable but curable disease. Cancer. 2006;107(9):2298-2306.

14. Harrison ML, Hoskins P, du Bois A, et al. Small cell of the ovary, hypercalcemic type - analysis of combined experience and recommendation for management. A GCIG study. Gynecol Oncol. 2006;100(2):233-238.

15. Senekjian EK, Weiser PA, Talerman A, Herbst AL. Vinblastine, cisplatin, cyclophosphamide, bleomycin, doxorubicin, and etoposide in the treatment of small cell carcinoma of the ovary. Cancer. 1989;64(6): $1183-1187$

16. Tewari K, Brewer C, Cappuccini F, Macri C, Rogers LW, Berman ML. Advanced-stage small cell carcinoma of the ovary in pregnancy: longterm survival after surgical debulking and multiagent chemotherapy. Gynecol Oncol. 1997;66(3):531-534.

17. Wallbillich JJ, Nick AM, Ramirez PT, Watkins JL, Euscher ED, Schmeler KM. Vinblastine, cisplatin, cyclophosphamide, bleomycin, doxorubicin, and etoposide (VPCBAE) in the management of three patients with small-cell carcinoma of the ovary. Gynecol Oncol Case Rep. 2012;2(2):58-60.

18. Walker NH, Sabanli M, Sykes PH, Russell P, Perez D. Successful reproductive outcome following treatment of advanced small cell carcinoma of the ovary. Gynecol Oncol Case Rep. 2012;2(3):115-117.

19. Bakhru A, Liu JR, Lagstein A. A case of small cell carcinoma of the ovary hypercalcemic variant in a teenager. Gynecol Oncol Case Rep. 2012;2(4):139-142.

20. Pautier P, Ribrag V, Duvillard P, et al. Results of a prospective doseintensive regimen in 27 patients with small cell carcinoma of the ovary of the hypercalcemic type. Ann Oncol. 2008;18(12):1985-1989.

21. Stephens B, Anthony SP, Han H, et al. Molecular characterization of a patient's small cell carcinoma of the ovary of the hypercalcemic type. J Cancer. 2012;3(1):58-66.

22. Karanian-Philippe M, Velasco V, Longy M, et al. SMARCA4 (BRG1) loss of expression is a useful marker for the diagnosis of ovarian smal cell carcinoma of the hypercalcemic type (ovarian rhabdoid tumor): a comprehensive analysis of 116 rare gynecologic tumors, 9 soft tissue tumors, and 9 melanomas. Am J Surg Path. 2015;39(9):1197-1205.

23. Jelinic P, Mueller JJ, Olvera N, et al. Recurrent SMARCA4 mutations in small cell carcinoma of the ovary. Nat Genet. 2014;46(5):424-426.

24. Ramos P, Karnezis AN, Craig DW, et al. Small cell carcinoma of the ovary, hypercalcemic type, displays frequent inactivating germline and somatic mutations in SMARCA4. Nat Genet. 2014;46(5):427-429. 


\section{Publish your work in this journal}

OncoTargets and Therapy is an international, peer-reviewed, open access journal focusing on the pathological basis of all cancers, potential targets for therapy and treatment protocols employed to improve the management of cancer patients. The journal also focuses on the impact of management programs and new therapeutic agents and protocols on

patient perspectives such as quality of life, adherence and satisfaction. The manuscript management system is completely online and includes a very quick and fair peer-review system, which is all easy to use. Visit http://www.dovepress.com/testimonials.php to read real quotes from published authors.

Submit your manuscript here: http://www.dovepress.com/oncotargets-and-therapy-journal 